\title{
MOSAICING: CURRENT STATUS
}

\author{
T.J. CORNWELL \\ NRAO, ${ }^{1}$ Socorro, New Mexico, 87801
}

\begin{abstract}
Over the past several years, there has been substantial progress both in algorithms for mosaicing and in our understanding of the effects of errors on mosaiced image. I describe the current state of knowledge in both these areas and also indicate where future improvements are likely to lie.
\end{abstract}

\section{INTRODUCTION}

For many areas of astronomy, the $\sim$ arcminute field of view allowed by moderate size antennas at millimeter wavelengths is too small. Objects often span many arcminutes or tens of arcminutes and therefore will not fit into one primary beam of an interferometric array. Single dishes can be used to image large fields at low resolution using standard methods but imaging with the high resolution attainable only by interferometry merits careful thought. In this paper, I use the name mosaicing to denote not just a single algorithm, but a systematic approach to this problem of imaging wide fields at high resolution using both single dishes and interferometers. This topic has been covered in a number of NRAO Millimeter Array memos (Cornwell 1984, 1987, 1988, Braun 1988, Holdaway 1990, 1992), in lecture notes (Cornwell 1989), and in a number of papers (Cornwell 1988, Mundy et al., 1988, Cornwell, Holdaway and Uson, 1993). Here I will summarize our current knowledge.

The algorithms described here are available in AIPS, in a development package SDE used internally at NRAO, and will be implemented in AIPS++.

\section{GOALS AND ASSUMPTIONS}

Our Goals for mosaicing are as follows:

- it should allow accurate imaging of a wide field of view spanning many primary beams at interferometric resolution,

- it should produce high imaging quality as measured by indicators such as on-source and off-source signal-to-noise ratio,

- it should be generalizable to other data such as, for example. that obtained by beam switching (Emerson et al., 1979).

\footnotetext{
${ }^{1}$ Associated Universities Inc. operates the National Radio Astronomy Observatory under National Science Foundation Cooperative Agreement No. AST-8814515
} 
- it should be computable with moderate resources,

- and it should be amenable to analysis.

We initially make the following Assumptions:

- The measurement equation is as follows. The visibility $V$ measured by an interferometer of baseline $u$ pointing at position $\mathbf{x}_{\mathrm{p}}$ is given by:

$$
V\left(\mathbf{u}, \mathbf{x}_{\mathrm{p}}\right)=\int A\left(\mathbf{x}-\mathbf{x}_{\mathrm{p}}\right) I(\mathbf{x}) e^{j 2 \pi \mathrm{u} \cdot \mathbf{x}} d \mathbf{x}
$$

A single dish is a simple special case, as is a beam-switched total power system.

- Visibility data can be collected for a range of pointing positions, $x_{p}$, spanning an object,

- We know the primary beam $A$ reasonably well (e.g. down to 1 st primary beam sidelobe). This may be relaxed subsequently but it is a realistic initial goal.

- The data are well-calibrated and free from significant errors. Again, this may be relaxed in more sophisticated work.

- Neither the image plane nor the Fourier plane sampling are necessarily complete. Special cases may arise when one or the other is good enough but we will not assume perfect sampling.

- The objects of interest have structure on most spatial scales. Thus the emission is more complicated than, for example, just a collection of a moderate number of point sources. This means that we desire accurate reconstruction of the visibility function over large regions of the Fourier plane.

\section{MOSAICING ALGORITHMS}

I will not describe the reasoning behind the development of the various algorithms for mosaicing since this is covered in the publications cited above. Instead, I will classify the algorithms according to the situation for which each one is designed.

Perfect coverage, single pointing If we ignore the effects of the dirty beam, then for a single pointing, the true sky can be recovered by dividing the dirty image $I^{D}$ by the primary beam.

$$
I^{\text {DIVISION }}(\mathbf{x})=\frac{I_{\mathbf{p}}^{D}(\mathbf{x})}{A\left(\mathbf{x}-\mathbf{x}_{\mathrm{p}}\right)}
$$

In AIPS this is performed by PBCOR. 
Perfect coverage, multiple pointings For multiple pointings, we can generalize the previous result by a least squares formalism to yield:

$$
I^{\mathrm{LSQ}}(\mathbf{x})=\frac{\sum_{\mathrm{p}} A\left(\mathbf{x}-\mathbf{x}_{\mathrm{p}}\right) w_{p} I_{\mathrm{p}}^{D}(\mathbf{x})}{\sum_{\mathrm{p}} A\left(\mathbf{x}-\mathbf{x}_{\mathrm{p}}\right)^{2} w_{p}}
$$

In AIPS this is performed by LTESS.

Limited coverage, multiple pointings If the sidelobes of the dirty beam are important then we can deconvolve each dirty image before combination. The most obvious drawback to this approach is that adjacent fields cannot help each other in the deconvolution.

$$
I^{\text {TIME-HONORED }}(\mathbf{x})=\frac{\sum_{\mathrm{p}} A\left(\mathbf{x}-\mathbf{x}_{\mathrm{p}}\right) w_{p} I_{\mathbf{p}}^{\text {CLEAN }}(\mathbf{x})}{\sum_{\mathbf{p}} A\left(\mathbf{x}-\mathbf{x}_{\mathbf{p}}\right)^{2} w_{p}}
$$

Limited but identical coverage If synthesized sidelobes are important and are roughly the same for all pointings then we can deconvolve an average dirty beam after linear combination. To a good approximation, the average PSF is given by:

$$
B^{\mathrm{LM}}(\mathbf{x})=B(\mathbf{x})\left[\frac{\sum_{\mathrm{p}} A_{\mathrm{p}}(\mathbf{x}) A_{\mathrm{p}}(0) w_{p}}{\sum_{\mathrm{p}} A_{\mathbf{p}}^{2}(\mathbf{x}) w_{p}}\right]
$$

We can therefore define an estimate of the sky obtained by solving the approximation convolution equation:

$$
I^{\mathrm{LSQ}}(\mathbf{x}) \approx B^{\mathrm{LM}}(\mathbf{x}) \otimes I^{\mathrm{LM}}(x)
$$

For the MMA compact configuration, $B^{\mathrm{LM}}$ has no zeroes in the Fourier transform and so linear deconvolution (e.g. Wiener filtering) can be used! In general, the Maximum Entropy Method (MEM, see e.g. Narayan and Nityananda, 1986) or CLEAN (Hogbom, 1974) could be used. We tend to call this approach linear mosaicing even though a non-linear deconvolution algorithm can be used in the final step.

Limited, non-identical coverage The very best scheme is to deconvolve all fields jointly, using MEM (or something like it) to find an image of the whole region which (a) fits all the measured data, and (b) has some desirable property such as maximal entropy. The joint deconvolution allows adjacent pointings to reinforce each other in the estimation of missing spacings. This is important because the deconvolution algorithms, such as MEM, are non-linear and work better with more complete Fourier plane coverage. In AIPS this is the VTESS/UTESS algorithm. It is also known as non-linear mosaicing.

Poor knowledge of primary beam We can relax one of the assumptions described above, namely that we know the primary beam well. Braun (1988) argued that instead of enforcing consistency between all pointings on all spacings, we should then use full non-linear mosaic only on the short, 
low $T_{B}$, baselines, and separate deconvolution on long, high $T_{B}$ spacings, merging answers in the Fourier plane. This reflects the fact that full-nonlinear mosaicing is often most useful for the short spacings, while for the longer spacings, the diameter of the array elements is small compared to the typical spacing between Fourier plane samples.

These algorithms are available in AIPS and are used routinely for reducing VLA and other array mosaicing observations. More sophisticated versions exist in SDE and are used for simulations and tests.

The computing costs vary:

Linear mosaicing : The costs lie principally in gridding, Fourier transformation and combination of the data from individual fields, followed by deconvolution of entire field. This is therefore roughly the same cost as deconvolving observations with much smaller element size.

Non-linear mosaicing : The costs lie also in gridding, Fourier transformation and combination of individual fields, but now repeated many times as iteration proceeds. This may seem expensive, but compared to separate non-linear deconvolution of the individual field, the increase in CPU time is only about $2-3$, if limited size transforms are used.

\section{UNDERSTANDING MOSAICING}

Mosaicing can be understood in a number of different and complementary ways. Perhaps the simplest is based upon a short paper by Ekers and Rots (1979) on a method of synthesizing the $0 \mathrm{~m}$ and $18 \mathrm{~m}$ spacings missing from WSRT observations. It is well-known that for a single dish, we can recover spacings $\pm D / \lambda$ around zero by scanning across an object and Fourier transforming the total power measurements with respect to scan position. The contribution of Ekers and Rots was to derive an analogous result for a two-element interferometer: namely that we can recover spacings $\pm D / \lambda$ around the nominal $B / \lambda$ by scanning across an object and Fourier transforming the measured visibility with respect to scan position. Cornwell (1988) derived a sampling theorem to show that we actually need samples only on a discrete grid of image plane points spaced $\Delta \mathbf{x}_{\mathrm{p}} \leq \frac{\lambda}{2 D}$. Although Ekers and Rots were interested only in the missing spacing problem, it is clear that this result can be used to derive spacings on a very fine grid in the Fourier plane. If we sample the visibility for a grid of pointing positions of side $N_{p}$ pointings, then we can recover spacings on grid $\Delta \mathrm{u} \sim \frac{D}{N_{p} \lambda}$ in Fourier plane. One nice way to understand this result is to note that, effectively, we have decreased the element size from $D$ to $\sim \frac{D}{N_{p}}$. We have therefore performed "element synthesis". The linear and non-linear mosaicing algorithms described above are more flexible than the Ekers and Rots scheme. Linear mosaicing can be seen as a optimum generalization of Ekers and Rots for many interferometers, while non-linear mosaicing can be regarded as Ekers and Rots for many interferometers plus some "glue" from the entropy term to help interpolate missing spacings. 


\section{ANALYSIS OF MOSAICING}

Cornwell, Holdaway and Uson (1993) have recently performed an extensive analysis of mosaicing. They used three methods to investigate properties of mosaicing:

Analysis $\Rightarrow$ basic understanding, first-order constraints on effects of errors such as noise, pointing, surface errors, etc. This is possible only for linear methods.

Simulations $\Rightarrow$ check basic understanding, provides detailed constraints on design. They produced an extensive simulation package capable of simulating many different types of error. To assess image performance, they developed measures of imaging quality. Simulations such as these are useful for checking analysis of the linear methods and for investigating non-linear methods.

Observational tests $\Rightarrow$ provide basic check of results, new types of error, the unexpected.

One of the great virtues of linear mosaicing is that simple error propagation can be used to derive the effects of various types of errors on the final image. Cornwell, Holdaway and Uson (1993) derive the following results for the noise level $\sigma$ and the on-source signal-to-noise ratio $\Lambda$.

\section{Additive errors}

$$
\sigma_{I^{\mathrm{LM}}}=\frac{\sqrt{\sum_{p} A_{p}^{2}(\mathbf{x}) \sigma_{I_{p}^{D}}^{2}}}{\sum_{p} A_{p}^{2}(\mathbf{x})}
$$

\section{Primary beam errors}

$$
\Lambda^{P B E} \cong \frac{\sum_{p} A_{p}^{2}(\mathbf{x})}{\sqrt{\sum_{p} \sigma_{A_{p}}^{2} A_{p}^{2}(\mathbf{x})}}
$$

In the limit in which the errors are totally correlated between pointings the on-source SNR is limited to:

$$
\Lambda^{P B E} \cong \frac{1}{\sigma_{A_{p}}}
$$

\section{Pointing errors}

$$
\Lambda^{P E}=\left[\frac{\sqrt{\sum_{p} A_{p}^{2}(\mathbf{x})}}{2 \sqrt{2 \ln (2)}}\right] \frac{\theta_{A}}{\sigma_{x}}
$$

Typically, this is $\sim \theta_{A} / \sigma_{x}$.

\section{Surface errors}

$$
\Lambda^{S E}=\frac{1}{2 \sigma_{s}^{2}}
$$


The dynamic range will be larger than the on-source signal-to-noise ratio by something like the inverse of typical synthesized beam sidelobe level.

These results show that good imaging quality relies upon pointing accuracy, surface accuracy, and primary beam knowledge. Further discussion of the exact requirements is given by Cornwell, Holdaway and Uson (1993).

\section{WHAT NEEDS MORE WORK?}

Although some of the algorithms described here have been around for almost ten years, mosaicing is still under-developed. At the moment, mosaicing observations are sufficiently painful to perform with today's millimeter wavelength arrays that relatively few mosaicing observations are made, and of those, few have the signal-to-noise ratio to merit full non-linear mosaicing. This will change as more elements are added to existing arrays and as faster telescopes, such as the NRAO MMA, are built. I expect that as people come to use mosaicing algorithms more and more, there will be pressure for improvements in a number of areas:

- We need better non-linear methods, such as a version of MEM in which the noise is treated as a free variable rather than estimated by the user. It may also be fruitful to try completely different algorithms such as the SDI CLEAN (Steer, Dewdney and Ito, 1984).

- Self-calibration can be incorporated more deeply into mosaicing. For example, for complex gain self-calibration it will pay to alternate selfcal iterations with mosaic iterations. Self-calibration of pointing errors will also be possible for sufficiently bright objects. Emerson (1991) has already demonstrated cross-calibration of array and single dish data, and Holdaway (private communication) demonstrated an algorithm for mosaicing with a priori known pointing errors.

- Both the assessment of data quality and editing of bad data are considerably more difficult for mosaicing observations since the approximate temporal continuity of the visibility function measured by an interferometric array no longer holds.

- The design of new interferometric arrays is strongly affected by the need for mosaicing (Cornwell, Holdaway and Uson, 1993). Also impacted are on-line systems, and telescope scheduling programs.

- Finally, mosaicing should be incorporated deep into new software systems such as AIPS++ rather than tacked on as in AIPS.

\section{ACKNOWLEDGMENTS}

The work summarized here was done in (very enjoyable) collaboration with Robert Braun, Mark Holdaway, and Juan Uson, for which I thank them. 


\section{REFERENCES}

Braun, R., 1988, NRAO millimeter array memo 54, Socorro, NM.

Cornwell, T.J., 1984, Millimeter Array Memo 32, Socorro, NM.

Cornwell, T.J., 1987, Millimeter Array Memo 42, Socorro, NM.

Cornwell, T.J., 1987, Millimeter Array Memo 43, Socorro, NM.

Cornwell, T.J., $A \mathcal{E} A$, 202, 316-321, 1988.

Cornwell, T.J., 1988, Millimeter Array Memo 44, Socorro, NM.

Cornwell, T.J., 1988, Millimeter Array Memo 45, Socorro, NM.

Cornwell, T.J., 1989, "Wide Field Imaging III: Mosaicing," in Proc. NRAO Third Synthesis Imaging Summer School, Socorro 1988, eds. R. A. Perley, F. R. Schwab, and A. H. Bridle, Astronomical Society of Pacific, pp. 277286.

Cornwell, T.J., Holdaway, M.A. and Uson, J.M., 1993, "Radio-Interferometric Imaging of Very Large Objects: Implications for array design", $A B A$ (in press), 1993.

Emerson, D. T., Klein, U., Haslam, C. G. T., 1979, $A \mathcal{E} A$, 76, 92

Emerson, D. T., 1991, In: Cornwell, T.J., and Perley, R.A. (eds.), Proc. IAU Col. 131, Radio Inteferometry: Theory, Techniques and Applications, ASP, Provo, Utah, 441.

Ekers, R. D., Rots, A. H., 1979, In: C. van Schooneveld (ed.), Proc. IAU Col. 49, Image Formation from Coherence Functions in Astronomy. D.Reidel, $\mathrm{p} 61$.

Högbom, J., 1974, ApJS, 15, 417

Holdaway, M. A., 1990, NRAO millimeter array memo 61, Socorro, NM.

Holdaway, M. A., 1992, NRAO millimeter array memo 73, Socorro, NM.

Holdaway, M. A., 1992, NRAO millimeter array memo 74, Socorro, NM.

Mundy, L. G., Cornwell, T. J., Masson C. R., Scoville, N. Z., Bååth, L. B., Johansson, L. E. B., 1988, ApJ, 325, 382

Narayan, R., Nityananda, R., 1986, ARA $\mathcal{E} A$, 24, 127

Steer D.G., Dewdney, P.E., and Ito, M.R., (1984), $A \& A, \mathbf{1 3 7}, 159-165$. 


\section{DISCUSSION}

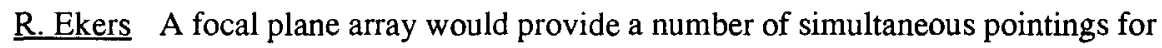
input to your mosaicing algorithms which would automatically give identical uv plane coverage. Would this be a significant advantage compared with sequential pointings?

T. Cornwell Yes. The advantages of focal plane array for mosaicing are numerous (a) speed, (b) coherence of eg. pointing errors for selfcalibration, (c) increased speed of pointing calibration, (d) improved total power measurement and (e) identity of $u, v$ coverage which in some cases can be exploited via the linear mosaicing algorithm described here.

R. Hills What range of timescales of pointing errors could be corrected by a method of the type you described?

T. Cornwell As in other forms of selfcalibration, it will be more useful for timescales a few times smaller than the calibration cycle. For the MMA, interferometric pointing is feasible in a few minutes, so we expect that pointing selfcalibration will be useful for minutes up to a hour or so. Timescales much shorter than the calibration cycle average out, leading to a broadened primary beam. 